Gut, 1971, 12, 693-701

\title{
An ultrastructural study of the liver in Indian childhood cirrhosis with particular reference to the structure of cytoplasmic hyaline
}

\author{
S. ROY, V. RAMALINGASWAMI, AND N. C. NAYAK \\ From the Department of Pathology, All-India Institute of Medical Sciences, New Delhi, India
}

SUMMARY Ultrastructural features of the liver in eight cases of Indian childhood cirrhosis, a fatal and elusive liver disease in India, have been described. Severe parenchymal damage, diffuse creeping fibrosis, and other light microscopic alterations, including the frequent presence of cytoplasmic hyaline, reported earlier are confirmed in the present study. The hyaline is predominantly comprised of tangled masses of fibrils and some electron-dense particles possibly derived from endoplasmic reticulum. The morphological features of this hyaline are identical to those of alcoholic hyaline described by Biava and Smuckler. Mitochondria do not seem to participate in the structural evolution of hyaline.

Indian childhood cirrhosis, an enigmatic and fatal liver disease, is prevalent among and almost exclusively limited to children in the Indian subcontinent. In this condition, profound morphological alterations occur in the hepatocyte (Liver Disease Sub-committee ICMR, 1955. Nayak and Ramalingaswami, 1970; Ramalingaswami and Nayak, 1970). While several reports describe the light microscopic appearance of the liver in this syndrome, ultrastructural study is limited to a single account given by Smetana, Hadley, and Sirsat (1961) which, however, is very brief and inconclusive. In our own studies of this condition we have recently drawn attention to the frequent presence of intracytoplasmic hyaline in the hepatocytes and emphasized the diagnostic and prognostic value (Nayak, Sagreiya, and Ramalingaswami, 1969). We have shown that the morphological, tinctorial, and histochemical characteristics of this hyaline are indistinguishable from those of the classical Mallory body of alcoholic livers. In spite of several studies, the nature and genesis of alcoholic hyaline itself still remains unclear (Norkin, Weitzell, Campagna-Pinto, MacDonald, and Mallory, 1960; Becker, 1961; Reppart, Peters, Edmondson, and Baker, 1963; Schaffiner, Loebel, Weiner, and Barka, 1963; Biava, 1964; Flax and Tisdale, 1964; Smuckler, 1968). The present communication is based on an ultrastructural study of the liver in Indian childhood cirrhosis with particular reference to the intracyctoplasmic hyaline. Received for publication 7 June 1971.

\section{Material and Methods}

Percutaneous needle biopsy specimens of the liver from eight cases of Indian childhood cirrhosis were collected for this study. The condition was diagnosed initially on clinical grounds and subsequently confirmed by light microscopic examination of the liver biopsy. One part of the tissue was divided into small blocks of $1 \mathrm{~mm}$ cube, fixed in $4 \%$ gluteraldehyde in phosphate buffer and postfixed in $1 \%$ osmium tetroxide buffered with veronal acetate. The tissues were then dehydrated in ascending grades of ethanol and embedded in Epon 812. Thin sections were cut with an L.K.B. automatic ultrotome, stained with uranyl acetate and lead citrate, and examined with a Hitachi HU-11A electron microscope. The other part of the tissue was fixed in neutral buffered formalin and processed for light microscopic examination in which routine haematoxylin-eosin stain and connective tissue strains were used.

\section{Results}

Light microscopic examination reveals characteristic features of Indian childhood cirrhosis as described by us earlier (Nayak et al, 1969; Nayak and Ramalingaswami, 1970; Ramalingaswami and Nayak, 1970) in all the eight cases. There is extensive and severe cytoplasmic damage in the hepatocytes accompanied by marked mesencymal reaction and creeping fibrosis. Parenchymal regeneration is 


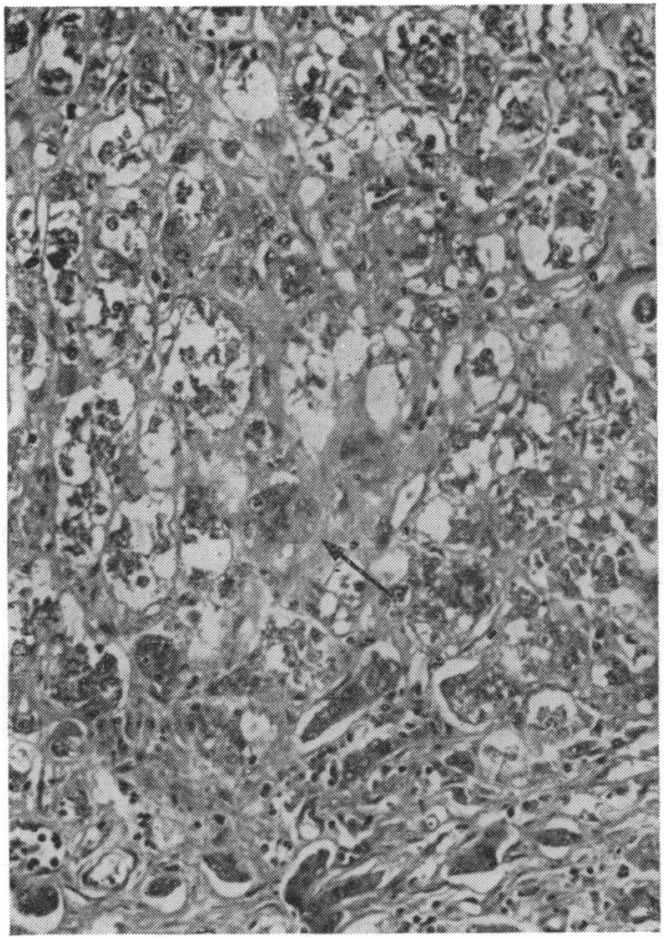

Fig. 1 Light microscopic picture of liver in Indian childhood cirrhosis showing marked degenerative changes of hepatocytes with ballooning of many cells, diffuse interstitial fibrosis, and ductular proliferation. Note the presence of Mallory's hyaline in many hepatocytes (arrow). Haematoxylin and eosin $\times 100$.

minimal or absent. Some of the hepatocytes present a puffed-up, ballooned appearance with the cytoplasmic contents preferentially aggregated at focal areas. Others are in an advanced stage of degeneration together with fuzziness and disruption of the cytoplasmic borders. Jrregular masses, tangles, and threads of a brightly eosinophilic material, the Mallory hyaline, are present in the cytoplasm of several hepatocytes (Fig. 1). These are frequently located in the perinuclear zone and vary considerably in size.

Mononuclear and neutrophilic cell infiltration, particularly around necrobiotic cells, and diffuse fibroblastic proliferation with collagen being laid down are prominent. Nodular regeneration is rare. Cholestasis, both cellular and canalicular, is frequently encountered.

Electron microscopically too severe degenerative change in the liver cells and diffusely scattered fibrosis are quite evident, as is the striking appearance of the hyaline deposits. Degenerative changes were fairly widespread involving most of the hepatocytes in all the eight cases. Hyaline deposition was frequent in six cases but was less frequent in the other two.

HY ALINE

Hyaline deposits usually appear in the perinuclear zone as fairly well defined masses of homogeneous, moderately electron-dense material (Fig. 2). There is, however, no limiting membrane separating them from the surrounding cytoplasm and at places the material appears to be merging with the adjacent cytoplasmic structures. In many areas in the zone immediately surrounding the hyaline, rough endoplasmic reticulum and groups of free ribosomes can be seen (Figs. 2-4). Some of the rough endoplasmic reticulum at this site appear to be fragmented and merging with the hyaline mass. At higher magnification the hyaline masses are found to be composed predominantly of fibrillary material admixed with fine granules (Figs. 4 and 5). The fibrillar component appears as fine, short membrane profiles, measuring approximately $80-130 \AA$ in diameter. They are irregularly distributed throughout the hyaline mass and are not orientated in any particular direction. The fibrils are generally straight profiles but at times they are found to be curved. Occasionally some of these fibrils are disposed in closely packed or even clumped masses. Scattered in the hyaline deposit are present numerous very fine granules of moderate electron density. Larger particles can also be seen either singly or in small groups. A few of these particles measure approximately 400-500 and appear to be glycogen, while others are smaller. measuring approximately $150 \AA$. and structurally resemble ribosome particles. In addition, the occasional vesicular structure and recognizable fragments of rough endoplasmic reticulum are noted (Figs. 3-5).

Apart from the presence of hyaline, other interesting changes are observed in the hepatocyte. Strikingly, many hepatocytes appear very pale and show large electron-lucent areas within the cytoplasm (Fig. 6). In such areas, the cytoplasmic organelles are absent or fewer in number and are rather loosely arranged. It is unlikely that these clear areas represent glycogen since in our sections, having been stained by lead, glycogen should have shown up as electron-dense particles. The same cells may show areas where the organelles, particularly the mitochondria, are present in large numbers and crowded together. In many other cells, irregular dilatation of the cisterns of rough endoplasmic reticulum is evident (Figs. 7 and 8). The dilatation is very marked in some places, resulting in distortion of the rough endoplasmic reticulum system with the formation of spaces of irregular contour into which projections of 


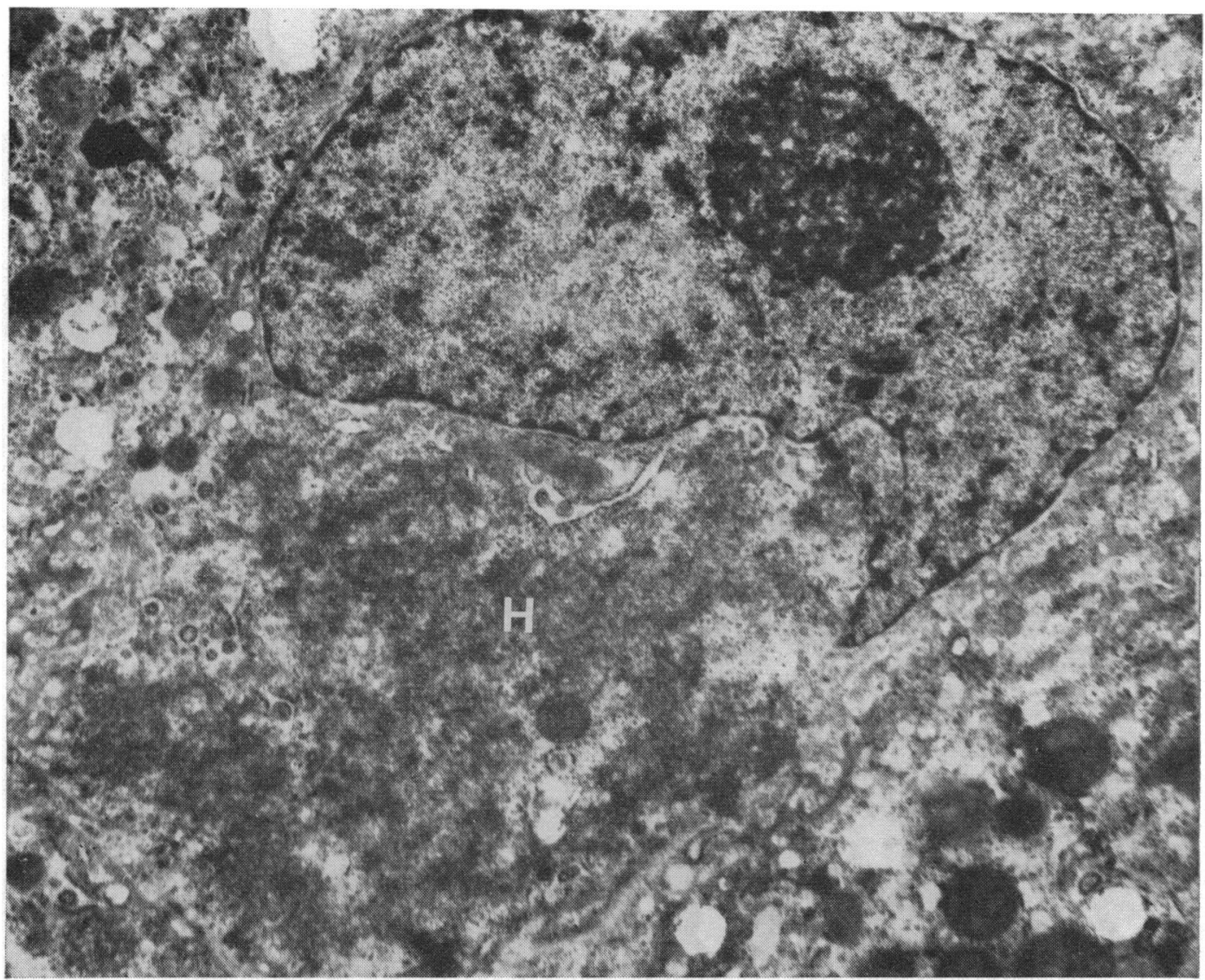

Fig. 2 Electron micrograph showing intracytoplasmic hyaline $(H)$ as an electron-dense mass within a hepatocyte. Note its perinuclear site and irregular outline. No limiting membrane is present. $\times 16,000$.

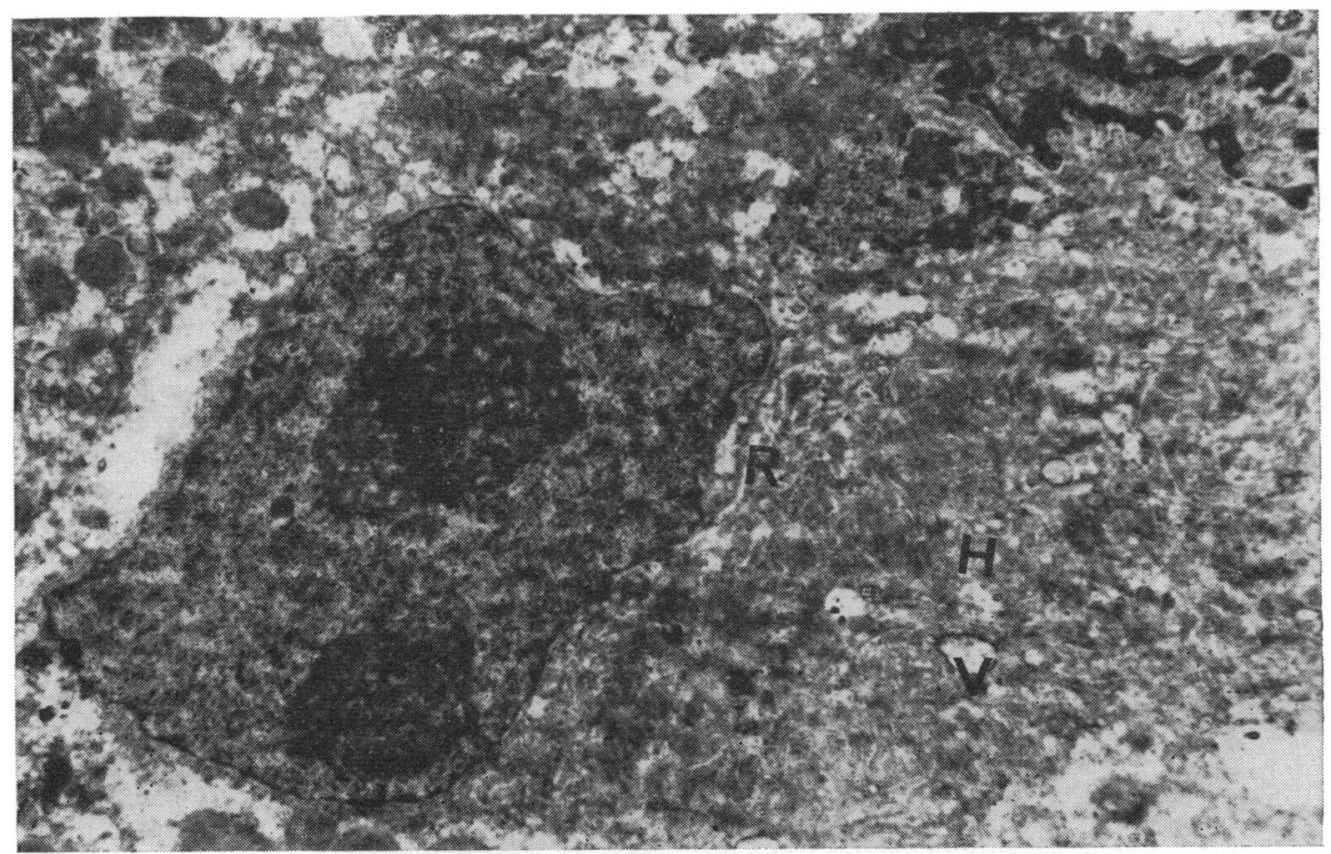

Fig. 3 Hepatocyte containing a hyaline mass $(H)$ within which recognizable cisternae of rough endoplasmic reticulum and a few vesicular structures $(V)$ are seen. Rough endoplasmic reticulum $(R)$ is also noted in the adjacent cytoplasm. $\times 8,000$. 


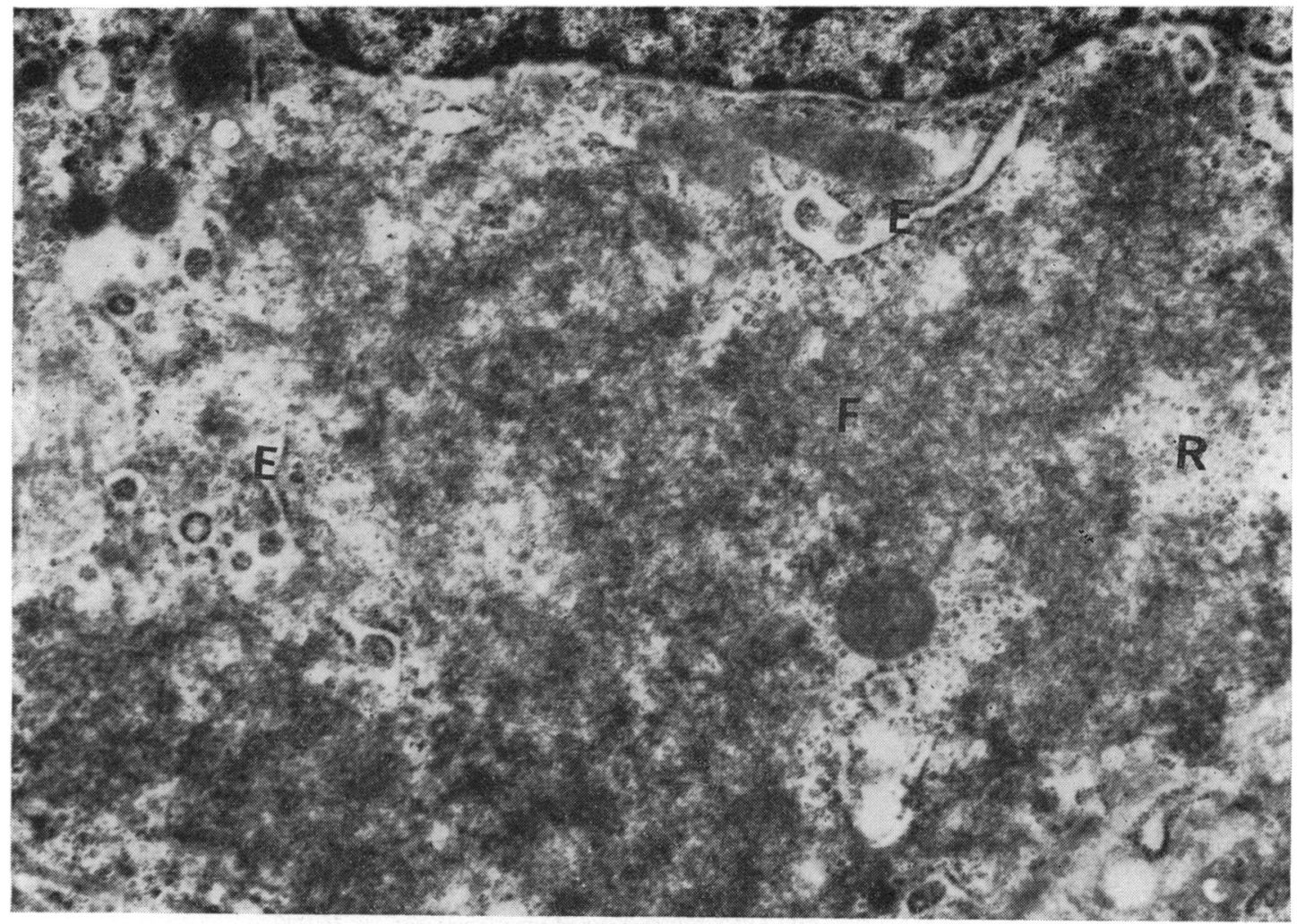

Fig. 4 Electron micrograph showing details of hyaline in Figure 2. The fibrillar nature of the material $(F)$ is apparent. Profiles of rough endoplasmic reticulum $(E)$ and free ribosomes $(R)$ are seen in the neighbourhood of the hyaline. $\times 30,000$.

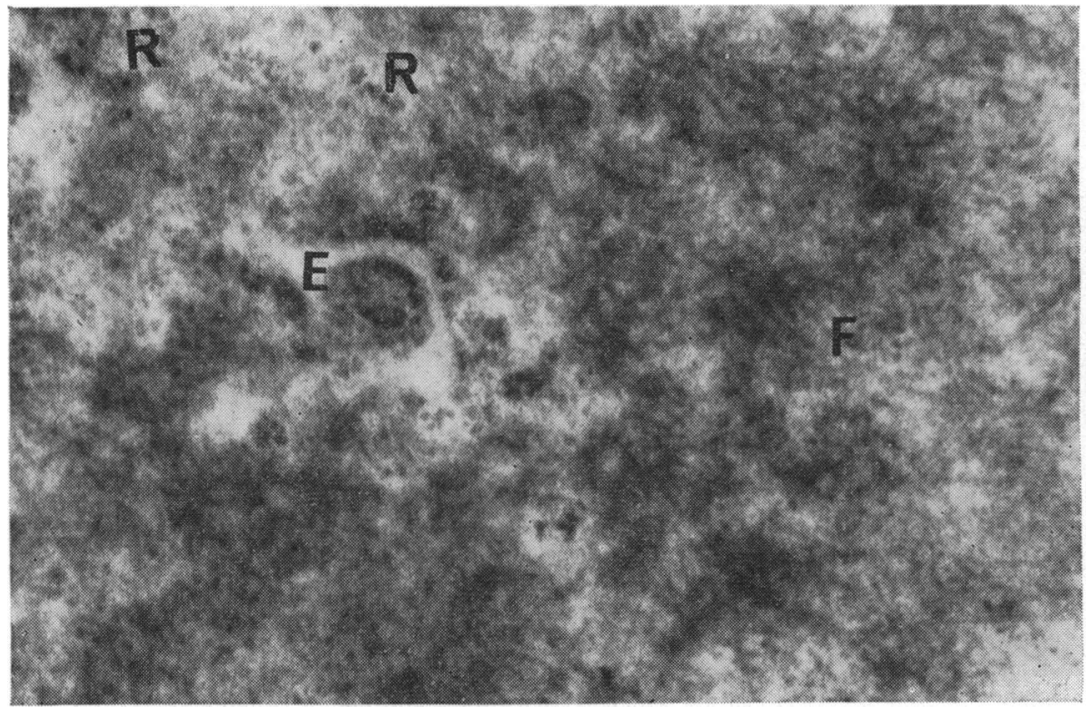

Fig. 5 Hyaline at higher magnification showing numerous fibrils $(F)$, fragmented rough endoplasmic reticulum $(E)$, and electron-dense particles $(R)$ probably representing free ribosomes. $\times 85,000$. 


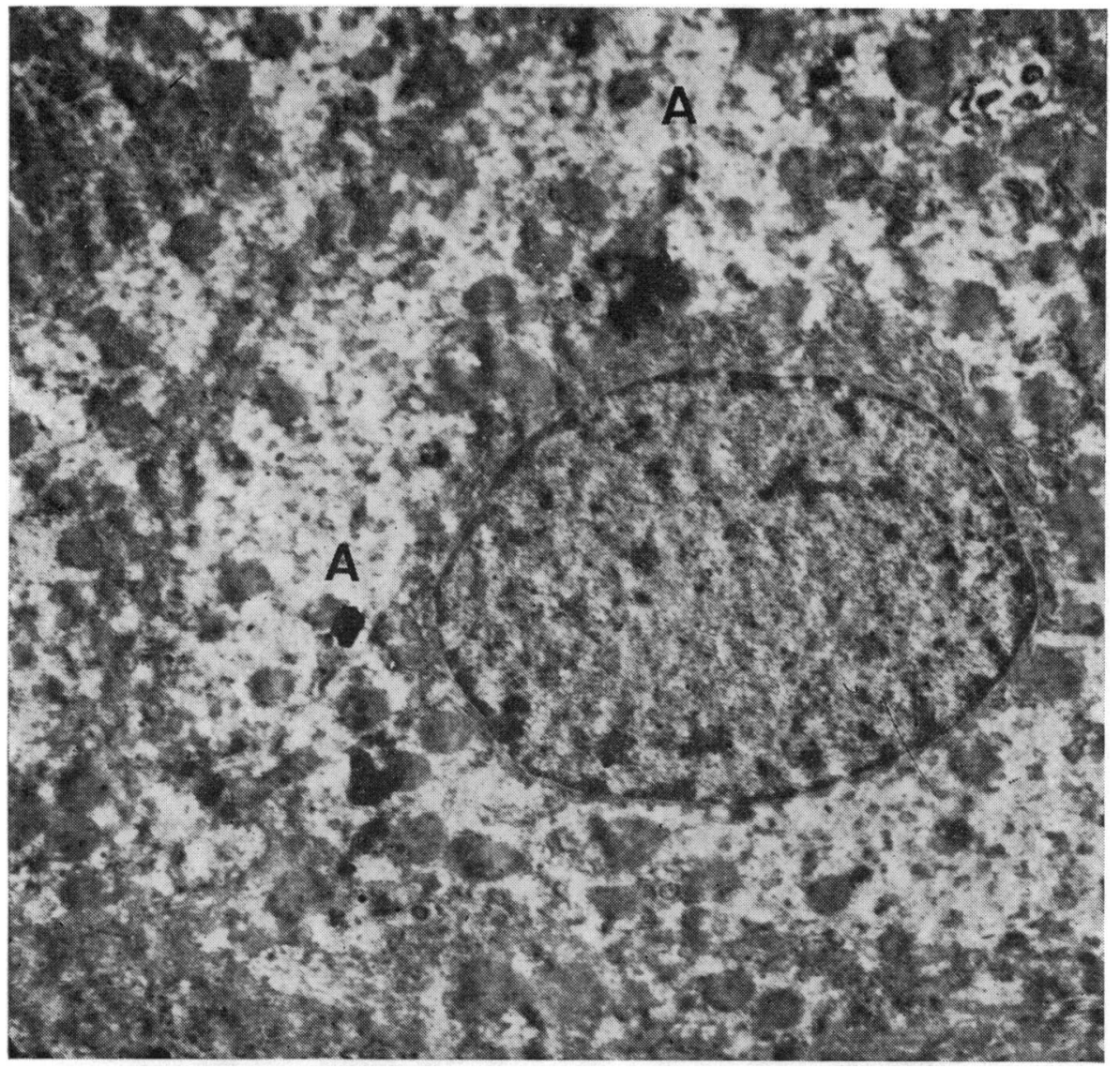

Fig. 6 Electron micrograph showing hepatocytes with large, pale electron-lucent areas $(A)$ in the cytoplasm. $\times 11,000$.

the distorted walls of rough endoplasmic reticulum are often seen. Mitochondria in hepatocytes showing advanced degeneration appear somewhat fuzzy with loss of clear definition of the membrane and cristae, though they can still be distinguished without difficulty. They do not appear to participate in the formation of cytoplasmic hyaline.

Another common abnormality is the presence of many cytoplasmic dense bodies of variable size, shape, structure, and electron density (Fig. 8). Many of these are membrane-bound and contain varying amount of membranous and/or granular material within them, while others have well defined outlines but without any definite membrane and contain either homogeneous or granular electrondense material. These structures are usually seen in areas of the cytoplasm undergoing obvious degenerative change and are considered to be cytolysomes resulting from focal cytoplasmic degeneration. Not infrequently irregular clumps of bile and lipofuscin pigments are also seen (Fig. 9). Of the cytoplasmic changes described above, pallor of the hepatocytes with large electron-lucent areas is the most frequent abnormality. This is followed by dilatation of cisterns of rough endoplasmic reticulum and the presence of electron-dense lysosomal bodies in that order of frequency.

The nuclei of most of the hepatocytes appear pale with much less electron density than in the normal liver cell nucleus. On rare occasion the cell junctions between two hepatocytes appear to be widened but no significant change is seen in the bile canaliculi. However, the microvilli of hepatocytes at the sinusoidal border are frequently shortened or absent with flattening of the cell membrane (Fig. 10). In an occasional liver cell the sinusoidal microvilli have exaggerated lengths and are thrown into tentaclelike processes. Frequently, collagen fibres are seen in close proximity to the cytoplasmic membrane of hepatocytes in the perisinusoidal area (Fig. 10). The 


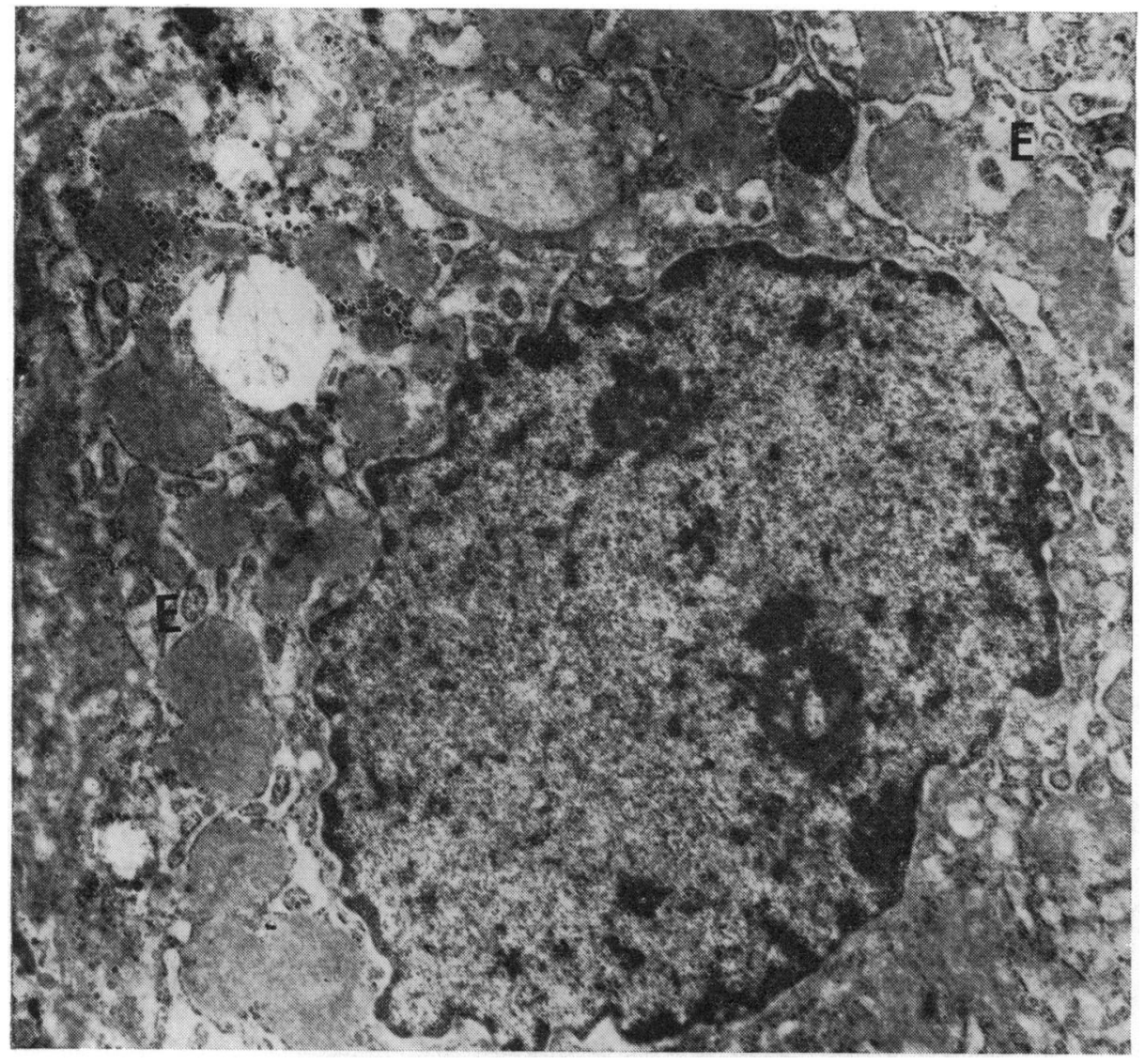

Fig. $7 A$

hepatocyte showing irregular dilatation of the cisternae of rough endoplasmic reticulum (E). In many areas projections of the wall of rough endoplasmic reticulum are seen in the dilated cisternae. $\times 16,500$.

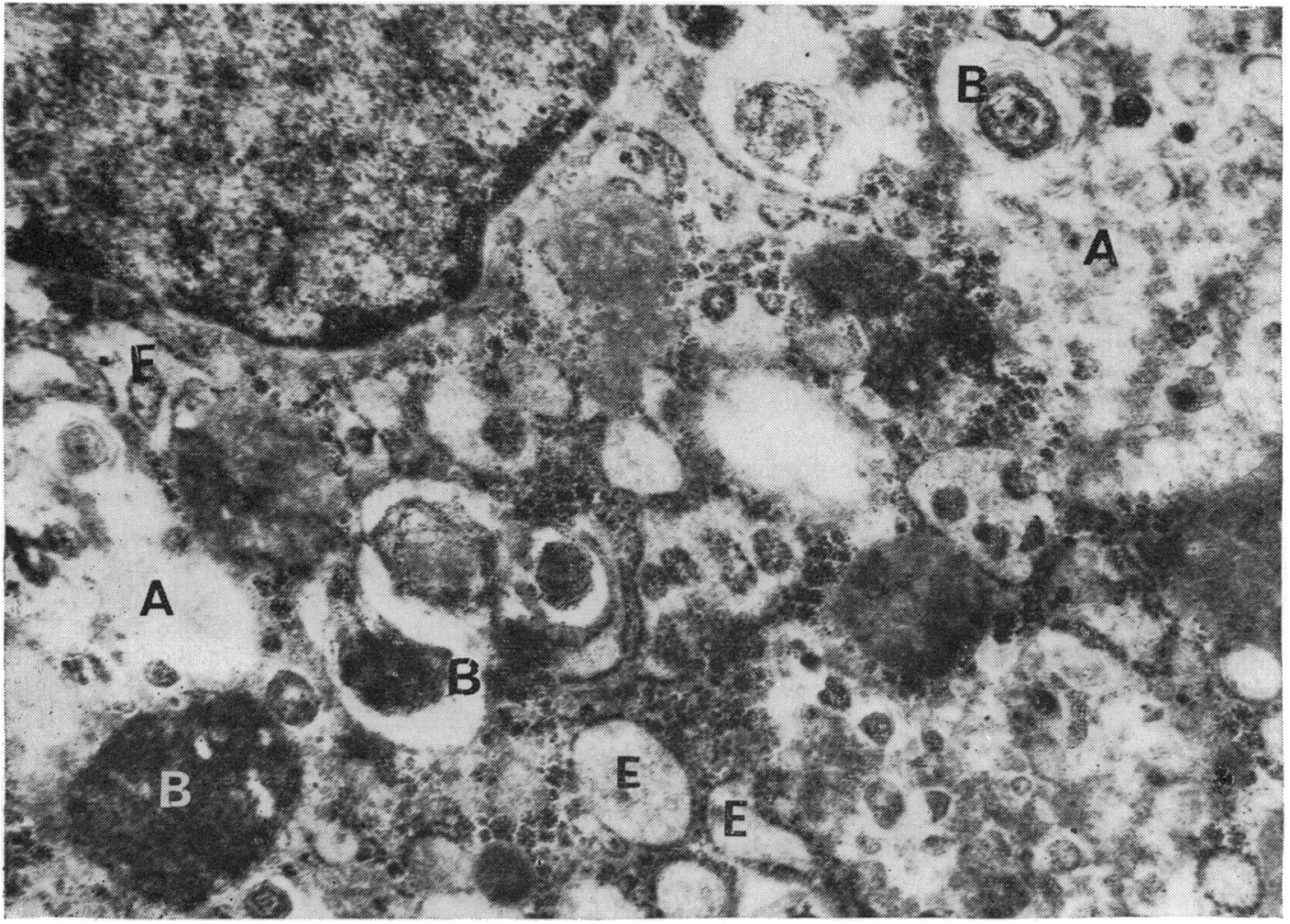

Fig. 8 A severely degenerated hepatocyte containing many electron-dense bodies $(B)$ of variable structure, electron-lucent areas $(A)$, and dilatation of cisternae of rough endoplasmic reticulum $(E) . \times 24,000$. 


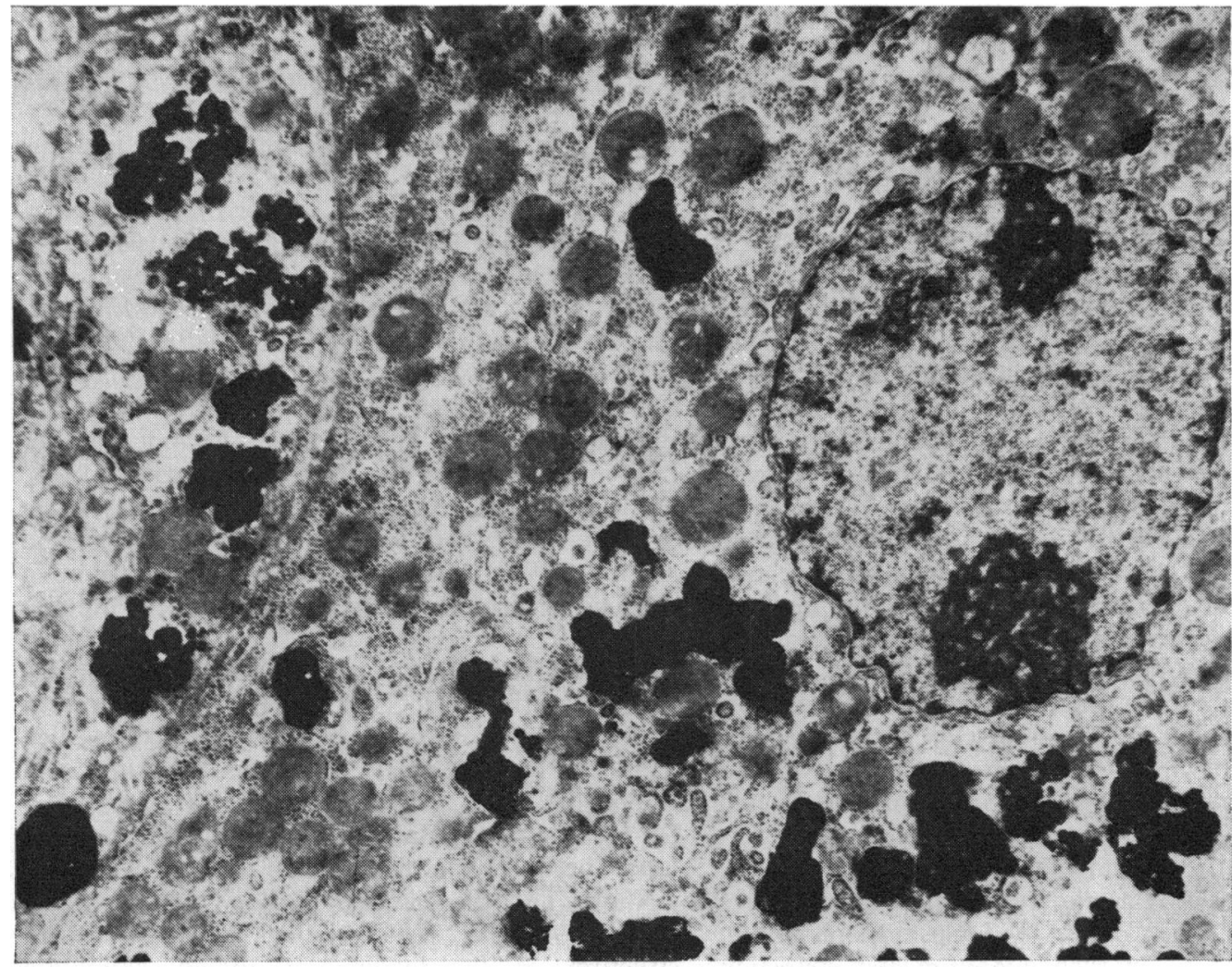

Fig. 9

Hepatocytes

containing

masses of

highly

electron-dense

material

possibly

representing

lipofuscin

and bile.

$\times 12,500$.

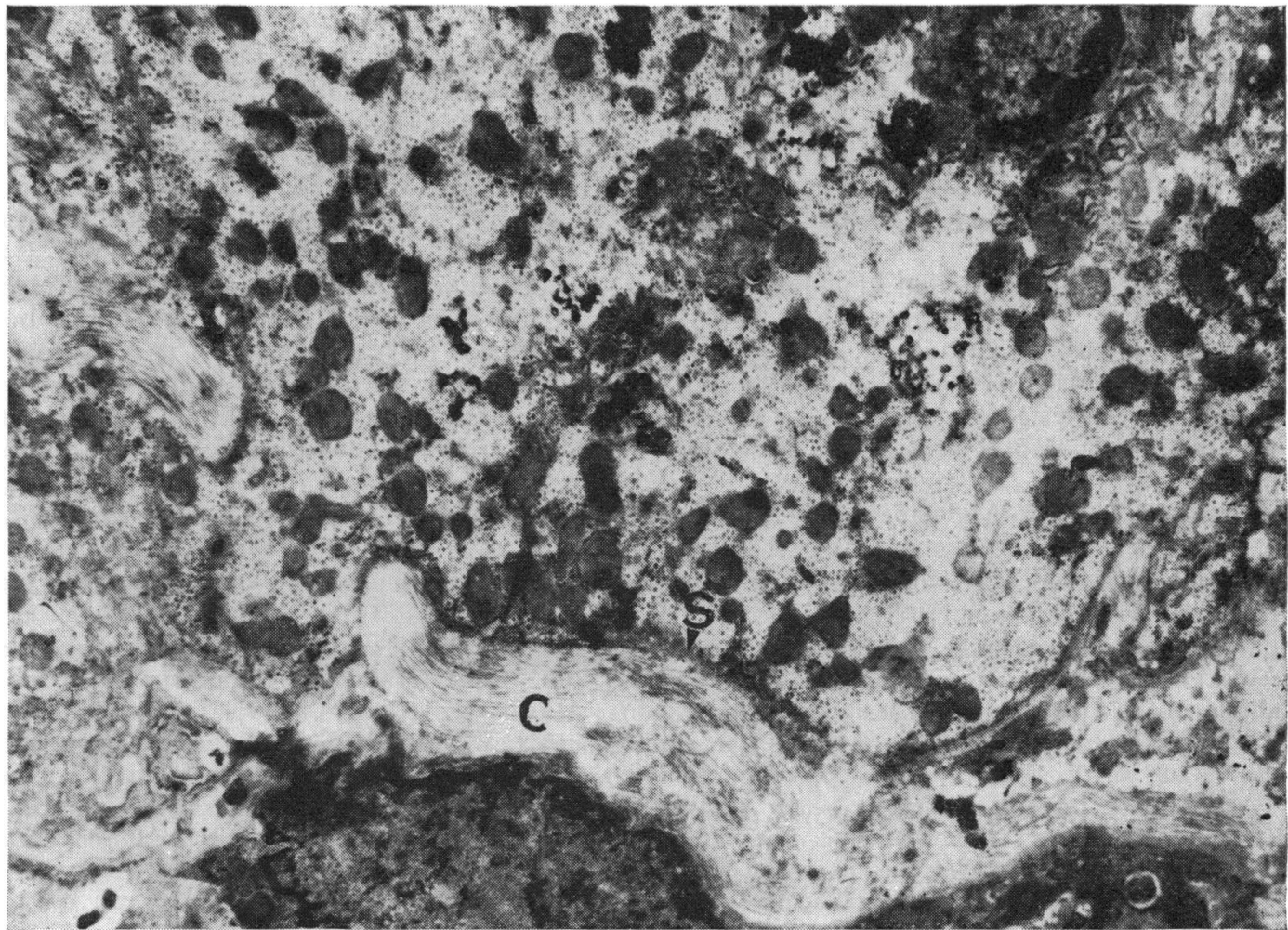

Fig. 10 Electron micrograph showing absence of microvilli of a hepatocyte in its sinusoidal border $(S)$ resulting in complete flattening of the cell membrane. There is a dense accumulation of collagen $(C)$ in the perisinusoidal region. $\times 8,000$. 
sinusoids in several areas are filled up and almost completely obliterated by hepatocytic debris, mesenchymal cells, and collagen fibres.

\section{Discussion}

The presence of cytoplasmic hyaline in hepatocytes as seen in histological preparations is a frequent finding in Indian childhood cirrhosis and may even be considered diagnostic in children between 1 and 5 years of age in this geographical region (Nayak et al, 1969). The present study shows that this hyaline consists predominantly of membrane structures together with particles having the general configuration of ribosomes. The fibrillar material may have originated from one or more of the several cytomembranes, particularly the endoplasmic reticulum. The frequent presence of rough endoplasmic reticulum and free ribosomes close to the hyaline masses, with which they often seem to merge, and the occurrence of fragments of recognizable rough endoplasmic reticulum within the hyaline support the idea that the hyaline is possibly derived mainly from the endoplasmic reticulum. Degenerative changes in the rough endoplasmic reticulum, characterized by marked dilatation and distortion of the cisterns in many parts of the hepatocytic cytoplasm, are also commonly observed. It is possible that these cytomembranes later disintegrate and coalesce to form masses of organoid structures visible as hyaline. Our own histochemical studies have earlier indicated that this hyaline, which is composed of lipoproteins and significant amounts of ribonucleoprotein particles, appears to be derived from endoplasmic reticulum (Nayak et al, 1969).

In their report on Indian childhood cirrhosis, Smetana and his colleagues (1961) mentioned briefly the ultrastructure of liver, including that of hyaline. On the basis of this rather poorly illustrated study, they suggested that the hyaline represented altered mitochondria together with aggregates of agranular reticulum. The single electron micrograph of hyaline shown in their paper seems to represent some intracytoplasmic pigment rather than hyaline. We find no evidence in our study to support the contention that mitochondria form a significant part of hyaline in Indian childhood cirrhosis. Aggregation of either normal or degenerated mitochondria, or, for that matter, of agranular reticulum within or close to the hyaline masses, is not seen in our material. In fact, in hepatocytes containing hyaline the mitochondria rarely show significant morphological alterations outside the areas of obvious cytoplasmic degradation. Such areas of focal cytoplasmic degeneration are usually situated some distance away from the areas containing hyaline.

It has been pointed out earlier that the hepatocytic hyaline in Indian childhood cirrhosis closely resembles Mallory's alcoholic hyaline (Nayak et al, 1969; Nyak and Ramalingaswami, 1970; Ramalingaswami and Nayak, 1970). The latter, however, is no longer considered specific for alcoholic liver disease and has been found in several other human diseases as well as in experimentally induced liver injury (Pappenheimer and Hawthorne, 1936; Baggenstoss and Stauffer, 1952; Hartcroft, 1958; Wilgram, 1959; Becker, 1961; Herdson, Garvin, and Jennings, 1964). Histochemical studies on hyaline in the liver of alcoholics have shown it to be composed chiefly of basic proteins of histone type combined with phospholipids and devoid of any carbohydrate moiety (Norkin et al, 1960; Becker, 1961). A recent study on the hyaline found in hepatomas has revealed identical histochemical characters (Norkin and Campagna-Pinto, 1968). We have also pointed out the morphological, tinctorial, and histochemical similarity between the hyaline of Indian childhood cirrhosis and the classical Mallory bodies (Nyak et al, 1969). It is interesting that the ultrastructure of the hyaline in Indian childhood cirrhosis, as observed in our present study, is in close agreement with that of alcoholic hyaline reported by Reppart et al (1963), Biava (1964), and Smuckler (1968). Biava (1964) for the first time presented evidence to suggest that the alcoholic hyaline of Mallory is composed almost entirely of ergastoplasmic membranes, revealing its triple layered structure and granular material resembling ribosomes. The ultrastructural features of the hyaline in our material from Indian childhood cirrhosis appear to be identical to those illustrated by Biava (1964) and by Smuckler (1968). The latter author, however, suggested that the physical and morphological characters of the alcoholic hyaline were unlike those of any one of the cytomembranes of the hepatocyte. $\mathrm{He}$ interpreted the particulate structures in the hyaline as a cross-sectional view of filaments rather than as ribosomes. It seems to us that it is very difficult to draw any firm conclusion about the nature of these fibrils and granules purely on the basis of morphological studies. The evidence from our earlier histochemical studies, however, supports our belief that the hyaline in Indian childhood cirrhosis is a product of or is derived from endoplasmic reticulum, a view identical to that expressed for alcoholic hyaline by Biava (1964). It is of further interest that in some forms of experimental liver injury hyaline bodies have been shown to be made up almost entirely of masses of endoplasmic reticulum (Bruni, 1960; Herdson et al, 1964). 
Some workers claim that the alcoholic hyaline is essentially derived from mitochondria. Flax and Tisdale (1964) are of the opinion that breakdown of mitochondrial and lysosomal elements of the cell and their subsequent coagulation gives rise to Mallory bodies. In their material the hyaline was membrane limited in its early phase of evolution and appeared to be derived from progressively degenerating mitochondria. We have seen no membrane around the hyaline in Indian childhood cirrhosis at any stage and mitochondrial elements are not closely incorporated in these structures. Biava's study (1964) on alcoholic hyaline also failed to reveal any mitochondrial participation. Porta and his colleagues in their experimental studies on chronic alcoholism in rats have repeatedly stressed that the hepatocytic hyaline in alcoholism represents large (megalomitochondria), deformed mitochondria (Porta and Gomez-Dumm, 1968). While the hyaline in their animals generally appears on light microscopy to be structurally different from the human alcoholic hyaline as well as from the hyaline in Indian childhood cirrhosis, it is also possible that the more irregular hyaline in their alcoholic rats may not show mitochondrial elements.

Other ultrastructural changes as observed in this study clearly depict the picture seen by light microscopic examination. Thus many cells showing large electron-lucent areas appear to represent the clear, ballooned cells. This is a very frequent alteration and is probably due to overhydration of the cells due either to some changes in membrane permeability allowing more fluid to get into the cells or to some alteration within the cell cytoplasm thereby changing its osmolarity so that it holds more water. The pale, vesicular nuclei on light microscopic examination appear as markedly dimished electron density of the nuclei. Large areas of cytoplasmic degeneration with the resulting formation of many cytolysosomes are also striking. In some portions of the cytoplasm mitochondrial aggregations represent the darker eosinophilic areas seen on light microscopy. The overall picture is one of varied and profound cell injury, largely of the cytoplasm, accompanied by mesenchymal cell reaction and fibrosis. The laying down of collagen in the perisinusoidal area and its insinuation through cell borders and cell junctions are similar to what has been described in other forms of cirrhosis.

The aetiology of Indian childhood cirrhosis is unknown. Nutritional factors do not appear to be operative and the role of hepatitis virus, toxin, genetic predisposition, and metabolic defects as yet remain uncertain (Ramalingaswami and Nayak, 1970; Nayak and Ramalingaswami, 1970; Chandra, 1970).

The authors wish to acknowledge help rendered by Dr Veena Chowla in providing the biopsies and by Mr Acchar Singh for technical assistance. Electron microscopy was done in the Department of Biophysics, All-India Institute of Medical Sciences.

\section{Reference}

Baggenstoss, A. H., and Stauffer, M. H. (1952). Post-hepatitic and alcoholic cirrhosis: clinicopathologic study of 43 cases of each. Gastroenterology, 22, 157-180.

Becker, B. J. P. (1961). The nature of alcoholic hyaline: A histochemical study. Lab. Invest., 10, 527-534.

Biava, C. (1964). Mallory alcoholic hyalin: a heretofore unique lesion of hepatocellular ergastoplasm. Lab. Invest., 13, 301-320.

Bruni, C. (1960). Hyaline degeneration of rat liver cells studied with the electron microscope. Lab. Invest., 9, 209-215.

Chandra, R. K. (1970). Immunological picture in Indian childhood cirrhosis. Lancet, 1, 537-540.

Flax, M. H., and Tisdale, W. A. (1964). An electron microscopic study of alcoholic hyaline. Amer. J. Path., 44, 441-453.

Hartroft, W. S. (1958). Intracellular ('Pseudo-alcoholic') hyaline in experiment al diet ary cirrhosis of rats and mice. (Abstr.) Amer. J. Path., 34, 603.

Herdson, P. B., Garvin, P. J., and Jennings, R. B. (1964). Fine structural changes in rat liver induced by phenobarbital. Lab. Inv st., 13, 1032-1037.

Indian Council of Medical Research (1955). Infantile cirrhosis of the liver in India. (Report of the Liver Disease Sub-committee.) Ind. J. Med. Res., 43, 723-749

Nayak, N. C., and Ramalingaswami, V. (1970). Indian childhood cirrhosis: pathologic features and etiologic considerations. In Pediatric Problems in Developing Countries, edited by $\mathbf{P}$. Tirumala Rao, pp. 229-240. Orient Longmans, Madras.

Nayak, N. C., Sagreiya, K., and Ramalingaswami, V. (1969). Indian childhood cirrhosis. The nature and significance of cytoplasmic hyaline of hepatocytes. Arch. Path., 88, 631-637.

Norkin, S. A., and Campagna-Pinto, D. (1968). Cytoplasmic hyaline inclusions in hepatoma: histochemical study. Arch. Path., 86, 25-32.

Norkin, S. A., Weitzel,'R., Campagna-Pi'nto, D., MacDonald, R. A. and Mallory, G. K. (1960). 'Alcoholic hyaline' in human. cirrhosis. Histochemical studies. Amer. J. Path., 37, 49-61.

Pappenheimer, A. M., and Hawthorne, J. J. (1936). Certain cytoplasmic inclusions of liver cells. Amer. J. Path., 12, 625-634.

Porta, E. A., and Gomez-Dumm, C. L. A. (1968). A new experimental approach in the study of chronic alcoholism. I. Effects of high alcohol intake in rats fed a commercial laboratory diet. Lab. Invest., 18, 352-364.

Ramalingaswami, V., and Nayak, N. C. (1970). Liver disease in India. In Progress in Liver Disease, edited by $\mathrm{H}$. Popper and F. Scheyfner, vol. 3, pp. 222-235. Grune and Stratton, New York.

Reppart, J. T., Peters, R. L., Edmondson, H. A., and Baker, R. F. (1963). Electron and light microscopy of sclerosing hyaline necrosis of the liver: the development of alcoholic hyaline bodies. Lab. Invest., 12, 1138-1153.

Schaffner, F., Loebel, A., Weiner, H. A., and Barka, T. (1963). Hepatocellular cytoplasmic changes in acute alcoholie hepatitis. J. Amer. med. Ass., 183, 343-346.

Smetana, H. F., Hadley, G. G., and Sirsat, S. M. (1961). Infantile cirrhosis. An analytical review of the literature and a report of 50 cases. Pediatrics, 28, 107-127.

Smuckler, E. A. (1968). The ultrastructure of human alcoholic hyalin. Amer. J. clin. Path., 49, 790-797.

Wilgram, G. F. (1959). Experimental Laennec type of cirrhosis in monkeys. Ann. intern. Med., 51, 1134-1158. 